\title{
The Third Way of Thermal-Electric Conversion beyond Seebeck and Pyroelectric Effects
}

\author{
Jie Ren* \\ Theoretical Division, Los Alamos National Laboratory, Los Alamos, New Mexico 87545, USA
}

(Dated: June 25, 2021)

\begin{abstract}
Thermal-electric conversion is crucial for smart energy control and harvesting, such as thermal sensing and waste heat recovering. So far, people are aware of only two ways of direct thermal-electric conversion, Seebeck and pyroelectric effects, each with distinct working conditions and limitations. Here, we report the third way of thermal-electric conversion beyond Seebeck and pyroelectric effects. In contrast to Seebeck effect that requires spatial temperature difference, the-third-way converts the time-dependent ambient temperature fluctuation into electricity, similar to the behavior of pyroelectricity. However, the-third-way is also distinct from pyroelectric effect in the sense that it does not require polar materials but applies to general conducting systems. We demonstrate that the-third-way results from the temperature-fluctuation-induced dynamical charge redistribution. It is a consequence of the fundamental nonequilibrium thermodynamics and has a deep connection to the topological phase in quantum mechanics. The findings expand our knowledge and provide new means of thermal-electric energy harvesting.
\end{abstract}

About 90 percent of the world's energy is utilized through heating and cooling, which makes energy waste a great bottleneck to the sustainability of any modern economy [1]. In addition to developing new technology of smart heat control [2], the global energy crisis can be alleviated by recovering the wasted thermal energy. In view of the inconvenient truth that more than $60 \%$ of the energy utilization was lost mostly as wasted heat [3], harvesting the thermal energy becomes critical to provide a cleaner and sustainable future [1].

Thermal-electric energy harvesting mainly relies on two principles: Seebeck effect (Fig. 1A) and pyroelectric effect (Fig. 1B). The Seebeck effect utilizes the spatial temperature difference between two sides of materials to drive the diffusion of charge carriers so as to convert heat into electricity $[4,5]$. Besides recovering waste heat, Seebeck effect with its reciprocal has wide applications of cooling, heating, power generating [6] and thus has revitalized an upsurge of research interest recently $[7,8]$. However, when the ambient temperature is spatially uniform, we have to resort to the pyroelectric effect [9], which utilizes the time-dependent temperature variation to convert heat into electricity but is restricted to pyroelectric materials [10]. This is due to the fact that the temporal temperature fluctuation modifies the spontaneous polarization of polar crystals, which consequently redistributes surface charges and produces temporary electric current $[11,12]$. In addition to thermal-electric energy harvesting [13-17], pyroelectric effect has widespread applications in long-wavelength infrared sensing, motion detector, thermal image [11], and even nanoscale printing [18].

However, since Seebeck and pyroelectric effects have been known for quite a long time, one cannot help wondering: Does Nature only offer us these two means for thermal-electric energy harvesting? Does any new principle of thermal-electric conversion exist beyond them? The Seebeck effect has been known for almost 200 years [19], and the pyroelectric effect, named in 1824 [20], can be even traced back to 314BC [11]. Now, it is time to think "out of the box", as advocated by Arun Majumdar [21].

In this work, we describe a third way of thermal-electric en- (a)

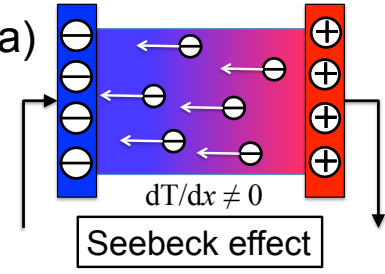

(b)



(d)



FIG. 1. Comparison of different thermal-electric conversion ways. (A) Seebeck effect converts the spatial temperature difference $(d T / d x \neq 0)$ to electricity by driving the thermal diffusion of charge carriers from hot to cold. (B) Pyroelectric effect converts the temporal temperature variation $(d T / d t \neq 0)$ to temporary electric current because variations of the temperature-dependent polarization in polar materials redistribute the surface charges. The typical behavior is similar to (D), see also Refs. [15-17]. (C) Distinctly, the-third-way produces electric current from the time-dependent temperature fluctuation in contrast to Seebeck effect, and it does not require polar materials but applies to general conducting system in contrast to pyroelectric effect. (D) The typical behavior of temperature-fluctuationinduced temporary electric current by the-third-way. It is in a similar manner as that of pyroelectric effect, see also Refs. [15-17].

ergy harvesting beyond Seebeck effect and pyroelectric effect. This third way converts the time-dependent ambient temperature fluctuation into electricity (Fig. 1, C and D), thus in contrast to Seebeck effect that requires spatial temperature difference. The third way is also distinct from pyroelectric effect in the sense that it does not require polar materials but applies to general conducting systems, although they produce the electricity in a similar manner (Fig. 1D). This third way of thermal-electric conversion is a consequence of the fundamental thermodynamics far from equilibrium. It results from the dynamical charge redistribution through the temperature 
(A)
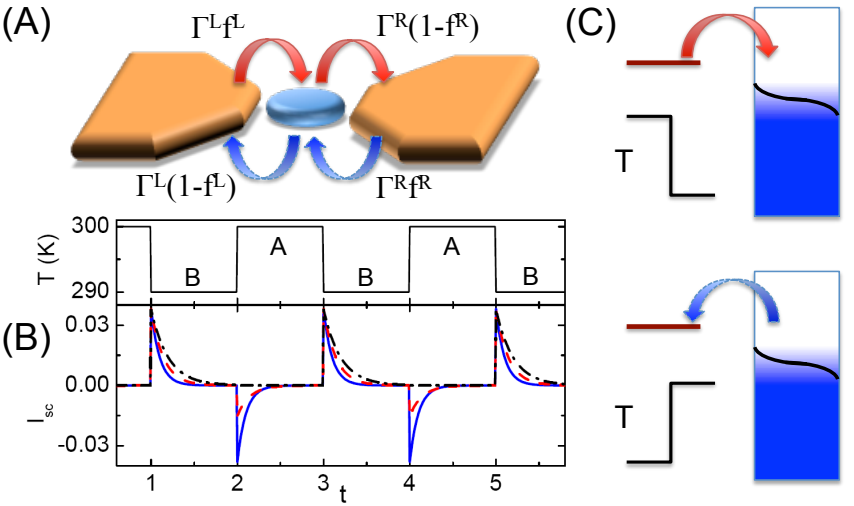

FIG. 2. The principle of the-third-way of thermal-electric conversion from the ambient temperature fluctuation. (A) Sketch of the single-level nanodevice and its charge transfer dynamics. (B) Temperature-fluctuation-induced temporary electric current. Temperature varies between $T_{A}=300 \mathrm{~K}$ and $T_{B}=290 \mathrm{~K}$. Other parameters are $\varepsilon_{0}=40 \mathrm{meV}, \Gamma_{A}^{L}=\Gamma_{B}^{R}=5, \Gamma_{A}^{R}=\Gamma_{B}^{L}=5$ (solid), 2 (dash), 0 (dash dot). The latter two cases show that the asymmetric system-lead coupling change rectifies the ac current. (C) Schematic illustration of the electron transfer through the right lead under temperature variation: when temperature decreases, the electron transfers from the central level to the lead; while when temperature increases, the electron is injected from the lead to the central level.

fluctuation and has a deep connection to the topological phase in quantum mechanics.

To illustrate the-third-way, we consider a typical nanodevice with only one electron level, as shown in Fig. 2A. Although we choose the single-level system for demonstration due to the simplicity, it is worth emphasizing that the following discussions can be readily generalized to bulk systems. The single-level nanodevice also has the great advantage of scalability and tunability, and it has many realizations such as single molecular junction and quantum dot system that have shown promise for energy applications [22-25]. The transfer kinetics is described as follows [26, 27]: An electron can hop from the $v(=L, R)$ lead into the single level with rate $\Gamma^{v} f^{v}$; When the level is occupied by an electron, the electron can escape to the $v$ lead with rate $\Gamma^{v}\left(1-f^{v}\right)$. Here $f^{v}=\left[e^{\left(\varepsilon_{0}-\mu^{v}\right) /\left(k_{B} T\right)}\right]^{-1}$ denotes the Fermi-Dirac distribution at the $v$ lead with $\varepsilon_{0}$ being the energy of the single level. $\Gamma^{v}$ is proportional to the system-lead coupling and the density of state of the lead $v$ at $\varepsilon_{0}$, so that $\Gamma^{v}$ is generally temperaturedependent $[28,29]$. Periodic temperature fluctuation is imposed on the whole system, following Refs. [15-17]. Without loss of generality, we adopt the square-wave fluctuation that is convenient for theoretical analysis and can be decomposed as linear superpositions of sine functions [30]. As such, the ambient temperature switches between high temperature $T_{A}$ and low temperature $T_{B}$, each with duration time $T_{p}$. Accordingly, the system's evolution can be divided into two ensembles, of which the parameters are denoted with the corresponding superscript A and B, respectively.

Without doubt, in either of two ensembles the system is in equilibrium with a homogenous temperature, which as is well known can not produce electric current. However, as we will show in the following the electric current indeed emerges with the help of temperature fluctuations, because switching between two equilibrium ensembles ultimately makes the whole evolution out of equilibrium. We set $\mu^{v}=0$ so that $f_{A, B}^{L}=f_{A, B}^{R}=f_{A, B}$ and focus on the short-circuit current through the right lead. Straightforward calculations lead us to the analytic expressions of temporary electric currents:

$$
I_{s c}=\left\{\begin{array}{l}
-\Gamma_{A}^{R}\left(f_{A}-f_{B}\right) \frac{1-e^{-K_{B} T_{p}}}{1-e^{-\left(K_{A}+K_{B}\right) T_{p}}} e^{-K_{A} \tau_{A}} \\
\Gamma_{B}^{R}\left(f_{A}-f_{B}\right) \frac{1-e^{-K_{A} T_{p}}}{1-e^{-\left(K_{A}+K_{B}\right) T_{p}}} e^{-K_{B} \tau_{B}},
\end{array}\right.
$$

where $K_{u}=\Gamma_{u}^{L}+\Gamma_{u}^{R}$ and $\tau_{u}=\bmod \left(t \in u, T_{p}\right)$ with $u=A, B$. Results are displayed in Fig. 2B, which shows consistency with the thermal-electric generation of pyroelectric effect in a similar manner [15-17].

The principle of the-third-way of thermal-electric conversion can be understood as follows (see also Fig. 2C): Before the temperature change, the central system and the lead have built an equilibrium, thus no net charge transfer. However, when ambient temperature fluctuates to lower one, less electrons will populate above the lead's Fermi level since the thermal broadening will shrink. As such, the balance is broken and the electron tends to transfer from the central system to the lead and then relax to a new equilibrium. When temperature fluctuates back to higher one, more electrons will populate above the lead's Fermi level due to the thermal broadening. Thus, the redundant conduction electron in the lead will transfer to the central system, trying to build a new balance. Therefore, even without polar materials, the-third-way is able to generate electric current from the ambient temperature fluctuation in general conducting system, which is intrinsic to the nonequilibrium thermodynamics. Although with different principles, considering the similar electric current generating behaviors between the-third-way and pyroelectric effect [1517], we may use a full-wave bridge circuit to rectify the ac current [16], which also can be achieved by the asymmetric system-lead coupling change (Fig. 2B).

We have shown that the-third-way of thermal-electric conversion is a consequence of the fundamental thermodynamics far from equilibrium. It results from the dynamical charge redistribution through the temperature fluctuation. In below, we are going to unravel its deep connection to the topological phase in quantum mechanics. The evolution of the system can be described by a Schrödingier equation in imaginary time, that is, a twisted master equation with the counting field $\chi$ :

$$
\frac{d}{d t}\left|\Psi_{\chi}(t)\right\rangle=\hat{H}(\chi, t)\left|\Psi_{\chi}(t)\right\rangle
$$

where $\left|\Psi_{\chi}(t)\right\rangle=\sum_{q=-\infty}^{\infty} e^{i q \chi}\left[p_{0}(q, t), p_{1}(q, t)\right]^{\mathrm{T}}$ with $p_{0 / 1}(q, t)$ denoting two joint probabilities that at time $t$ the single level is empty or occupied by an electron while there have been $q$ electrons transferred into the right lead. The transfer operation $\hat{H}$ is reminiscent of the Hamiltonian but nonHermitian. In either $u=A$ or $B$ ensemble, $\hat{H}$ is expressed 
as: $\hat{H}_{u}=\left(\begin{array}{cc}-K_{u} f_{u} & \left(\Gamma_{u}^{L}+\Gamma_{u}^{R} e^{i \chi}\right)\left(1-f_{u}\right) \\ \left(\Gamma_{u}^{L}+\Gamma_{u}^{R} e^{-i \chi}\right) f_{u} & -K_{u}\left(1-f_{u}\right)\end{array}\right)$. Therefore, after $n$ fluctuation periods the evolution at time $t=2 n T_{p}$ is described by the characteristic function:

$$
Z_{\chi}=\left\langle\mathbf{1}\left|\left[e^{\hat{H}_{B} T_{p}} e^{\hat{H}_{A} T_{p}}\right]^{n}\right| \Psi_{\chi}(0)\right\rangle \sim e^{n \Phi(\chi)}
$$

with $\langle\mathbf{1}|=(1,1)$, which in turn generates the total electron transfer per period via the relation: $Q:=\partial \Phi(\chi) /\left.\partial(i \chi)\right|_{\chi=0}$ with the cumulant generating function defined as $\Phi(\chi):=$ $\lim _{n \rightarrow \infty} \frac{1}{n} \ln Z_{\chi}$.

At the slow switch limit (large $T_{p}$ ), the evolution at either ensemble $\hat{H}_{u=A, B}$ is dominated by its eigenmode, of which the eigenvalue $\lambda_{u}$ has the largest real part, with $\left|\psi_{u}\right\rangle$ and $\left\langle\varphi_{u}\right|$ the corresponding normalized right and left eigenvectors, such that $e^{\hat{H}_{u} T_{p}} \approx\left|\psi_{u}\right\rangle e^{\lambda_{u} T_{p}}\left\langle\varphi_{u}\right|$. Therefore, the characteristic function can be expanded as:

$$
Z_{\chi}(t) \approx e^{\left(\lambda_{A}+\lambda_{B}\right) n T_{p}}\left[\left\langle\varphi_{A} \mid \psi_{B}\right\rangle\left\langle\varphi_{B} \mid \psi_{A}\right\rangle\right]^{n} C .
$$

with $C=\left\langle\mathbf{1} \mid \psi_{B}\right\rangle\left\langle\varphi_{A} \mid \Psi_{\chi}(0)\right\rangle /\left\langle\varphi_{A} \mid \psi_{B}\right\rangle$ an unimportant coefficient. This expansion of $Z_{\chi}$ gives the cumulant generating function $\Phi(\chi)$, composed of two contributions: $Z_{\chi} \sim$ $e^{n \Phi(\chi)}=e^{n\left(\Phi_{\mathrm{dyn}}+\Phi_{\mathrm{top}}\right)}$, where

$$
\begin{aligned}
& \Phi_{\mathrm{dyn}}=\left(\lambda_{A}+\lambda_{B}\right) T_{p}, \\
& \Phi_{\text {top }}=\ln \left[\left\langle\varphi_{A} \mid \psi_{B}\right\rangle\left\langle\varphi_{B} \mid \psi_{A}\right\rangle\right] .
\end{aligned}
$$

Being reminiscent of the dynamic phase in quantum mechanics, we call $\Phi_{\mathrm{dyn}}(\chi)$ the dynamic phase contribution, which presents the average evolution as if the system evolves in either ensemble separately. As a consequence of the detailed balance at each equilibrium ensemble, the dynamic phase contribution to the electric-thermal conversion is always zero: $\partial \Phi_{\text {dyn }} /\left.\partial(i \chi)\right|_{\chi=0}=0$.

The second part $\Phi_{\text {top }}(\chi)$ however possesses a nontrivial topological phase interpretation, which is responsible for the temperature-fluctuation-induced electron transfer. Different from the Hermitian Hamiltonian in quantum mechanics, the twisted transfer operator $\hat{H}_{u}$ are non-Hermitian such that its left eigenvector $\left\langle\varphi_{u}\right|$ is not the Hermitian conjugate of the corresponding right eigenvector $\left|\psi_{u}\right\rangle$, but only biorhonormal with each other. Therefore, writing $\left\langle\varphi_{A} \mid \psi_{B}\right\rangle=$ $\left|\left\langle\varphi_{A} \mid \psi_{B}\right\rangle\right| e^{i \phi_{A B}(\chi)}$ and $\left\langle\varphi_{B} \mid \psi_{A}\right\rangle=\left|\left\langle\varphi_{B} \mid \psi_{A}\right\rangle\right| e^{i \phi_{B A}(\chi)}$ with angle $\phi_{A B}$ and $\phi_{B A}$ as the analogy of Pancharatnam's topological phase [31, 32], we have $\phi_{A B} \neq \phi_{B A}$ generally. This is different from from those in Hermitian quantum mechanics, where $\phi_{A B}$ is always equal to $\phi_{B A}$ [33]. Therefore, the electron number per period resulting from this topological phase contribution is obtained as:

$$
Q=\left.\frac{\partial \Phi_{\text {top }}}{\partial(i \chi)}\right|_{\chi=0}=\frac{\left(f_{A}-f_{B}\right)\left(\Gamma_{A}^{L} \Gamma_{B}^{R}-\Gamma_{B}^{L} \Gamma_{A}^{R}\right)}{K_{A} K_{B}} .
$$

The transferred electron number per period only depends on the topological phase properties of $\left\langle\varphi_{u}\right|$ and $\left|\psi_{u}\right\rangle$ and is independent on the period in the large $T_{p}$ limit, which is simi-

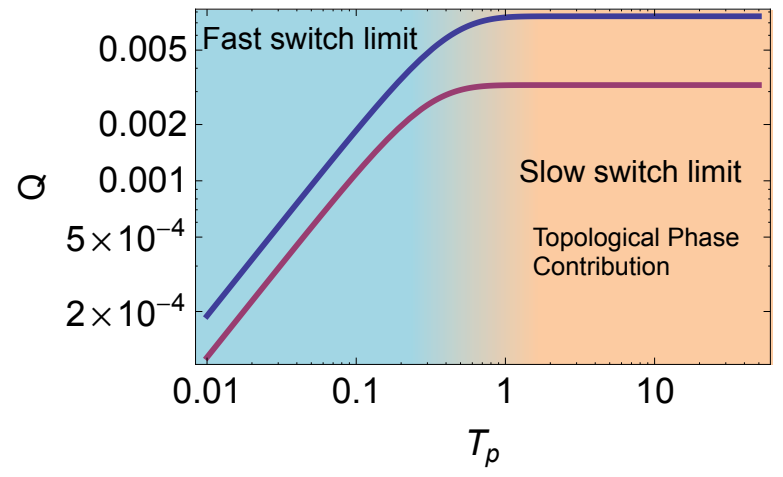

FIG. 3. The electron transfer generated per period by the-thirdway of thermal-electric energy harvesting. The thermal-electric conversion in the slow switch limit has an interpretation of topological phase, and gives the upper bound of the thermal-electric conversion per period. $\Gamma_{A}^{R}=\Gamma_{B}^{L}=0$ (upper curve), 2 (lower curve). Other parameters are the same as used in Fig. 2.

lar to the behavior governed by the adiabatic Berry phase effect $[34,35]$. However, we note that here the Pancharatnamlike topological phase contribution actually denotes a nonadiabatic process because of the abruptly discrete switching. The inner products $\left\langle\varphi_{B} \mid \psi_{A}\right\rangle$ and $\left\langle\varphi_{A} \mid \psi_{B}\right\rangle$ in the topological phase contribution characterize the asymmetric relaxation $\mathrm{dy}-$ namics from ensemble $\mathrm{A}$ to $\mathrm{B}$ and from ensemble $\mathrm{B}$ to $\mathrm{A}$. This nonequilibrium asymmetric relaxations between two equilibrium ensembles offer the nonzero net charge transfer per period.

At the fast switch limit (small $T_{p}$ ), we can approximate $\exp \left[\hat{H}_{B} T_{p}\right] \exp \left[\hat{H}_{A} T_{p}\right] \approx \exp \left[\left(\hat{H}_{A}+\hat{H}_{B}\right) T_{p}\right]$. Denote $\lambda_{A B}(\chi)$ as the dominated eigenvalue of the matrix $\left(\hat{H}_{A}+\right.$ $\left.\hat{H}_{B}\right)$, the solution is obtained as: $Q=T_{p} \partial \lambda_{A B} /\left.\partial(i \chi)\right|_{\chi=0}=$ $T_{p}\left(f_{A}-f_{B}\right)\left(\Gamma_{A}^{L} \Gamma_{B}^{R}-\Gamma_{B}^{L} \Gamma_{A}^{R}\right) /\left(K_{A}+K_{B}\right)$. In fact, the full exact solution can be similarly obtained as

$Q=\frac{\left(1-e^{-K_{A} T_{p}}\right)\left(1-e^{-K_{B} T_{p}}\right)\left(f_{A}-f_{B}\right)\left(\Gamma_{A}^{L} \Gamma_{B}^{R}-\Gamma_{B}^{L} \Gamma_{A}^{R}\right)}{\left(1-e^{-\left(K_{A}+K_{B}\right) T_{p}}\right) K_{A} K_{B}}$,

which is also seen as the result from integrating Eq. (1) within one period: $Q=\int_{0}^{2 T_{p}} d t I_{s c}(t)$. Clearly, the full exact solution in the small $T_{p}$ limit coincides with the fast switch result and in the large $T_{p}$ limit it reduces to the Pancharatnam-like topological-phase contribution Eq. (6). As shown in Fig. 3, the topological-phase contribution in the slow switching gives the upper limit of the thermal-electric conversion per period by the-third-way. Intuitively, $Q$ is proportional to $f_{A}-f_{B}$ that is in turn proportional to $T_{A}-T_{B}$, i.e., large temperature fluctuations produce more electricity. Moreover, Eq. (7) shows $Q$ is proportional to $\Gamma_{A}^{L} \Gamma_{B}^{R}-\Gamma_{B}^{L} \Gamma_{A}^{R}$. This indicates that the larger asymmetric coupling change $\Gamma_{A}^{L} / \Gamma_{A}^{R} \neq \Gamma_{B}^{L} / \Gamma_{B}^{R}$, the larger the electric generation. The largest asymmetry could be achieved by letting $\Gamma_{A}^{R}=0$ in one half period, and $\Gamma_{B}^{L}=0$ in the other half period, see Fig. 3, which corresponds to the anti-phase transfer: in ensemble A the electron transfers from the left lead to the central system that decouples with the right 
lead; in ensemble B, the central system decouples with the left lead and the electron transfers from the central part to the right lead. This picture also coincides with the largest rectification shown in Fig. 2B. If one prefers also the large average current per period $Q /\left(2 T_{p}\right)$, it is better to further choose $T_{p}$ near the boundary between fast and slow switch regimes, which is around $(\ln 10) / K_{u}$.

We now briefly estimate the short-circuit voltage, $V_{o c}$. Clearly, it will be achieved in the largest asymmetric case $\Gamma_{A}^{L} / \Gamma_{A}^{R} \gg \Gamma_{B}^{L} / \Gamma_{B}^{R}$. Let's further assume $+V_{o c} / 2$ is imposed on the left lead and $-V_{o c} / 2$ is imposed on the right one, and recall $T_{A}>T_{B}$. In the one half period, ensemble A, the electron transfers from the left lead to the central system, carrying entropy $\left(\varepsilon_{0}+e V_{o c} / 2\right) / T_{A}$; in the other half period, ensemble $\mathrm{B}$, the electron transfers from the central part to the right lead, releasing entropy $\left(\varepsilon_{0}-e V_{o c} / 2\right) / T_{B}$. The reversibility of the charge transfer at the open-circuit condition requires zero entropy production, so that $\left(\varepsilon_{0}+e V_{o c} / 2\right) / T_{A}=$ $\left(\varepsilon_{0}-e V_{o c} / 2\right) / T_{B}$. Therefore, we have the relation

$$
V_{o c}=2 \frac{\varepsilon_{0}}{e} \frac{T_{A}-T_{B}}{T_{A}+T_{B}}
$$

which is also confirmed numerically. It indicates that increasing the temperature fluctuation and meanwhile decreasing the average temperature can increase the open-circuit voltage that is upper bounded by $2 \varepsilon_{0} / e$.

Finally, we provide specific estimates for the-third-way converted electricity. The real coupling rates are usually at the scale of $\mathrm{GHz}$, indicating one electron hopping per nanosecond. We then can confer the unit $\mathrm{GHz}$ to the dimensionless couplings used for calculating Figs. 2 and 3. As such the peak current $I_{s c}=0.04$ in Fig. 2 corresponds to 6.4 pA, and the average current per period at $Q /\left(2 T_{p}\right) \approx 0.004$ in Fig. 3 corresponds to $0.64 \mathrm{pA}$. Considering the advantage of scalability in nanodevices, if we pack molecular quantum dots with spacing $50 \mathrm{~nm}$ between two metallic plate leads, we will have the packing density $4 \times 10^{10} / \mathrm{cm}^{2}$ that leads to the significant current density around the order of $0.1 \mathrm{~A} / \mathrm{cm}^{2}$. Also, the output voltage can be further optimized in practice, e.g. in the molecular junction the energy gap between the LUMO and the lead's Fermi level is around $\varepsilon_{0}=2 \mathrm{eV}[24,25]$, which compared to our used $\varepsilon_{0}=40 \mathrm{meV}$ can further improve the output voltage according to Eq. (8). Moreover, the temperature fluctuation in space can be as large as from $100 \mathrm{~K}$ to $500 \mathrm{~K}$, which will generate even better electricity by the-third-way.

Although there is widespread demand for both cost reductions and performance improvements, it is important at this stage to explore "out of the box" in principle. The-thirdway of thermal-electric conversion reported here is such an attempt. In contrast to Seebeck and pyroelectric effects, thethird-way is a consequence of the fluctuation-induced charge redistribution. It results from the fundamental nonequilibrium thermodynamics and has a deep connection to the topological phase in quantum mechanics. We believe that, as our ability to design and manipulate nano-systems is improving, thethird-way of thermal-electric conversion will provide the new means of energy harvesting to harness the ubiquitous temperature fluctuations in the world.

Jie Ren acknowledges the support from National Nuclear Security Administration of the U.S. DOE at LANL under Contract No. DE-AC52-06NA25396 through the LDRD Program.

*renjie@lanl.gov; jieustc@gmail.com

[1] S. Chu and A. Majumdar, Nature 488: 294-303 (2012).

[2] N. Li, J. Ren, L. Wang, G. Zhang, P. Hänggi, and B. Li, Rev. Mod. Phys. 84, 1045 (2012).

[3] Lawrence Livermore National Laboratory, "Estimated Energy Use in 2012: 95.1 Quads". https://flowcharts.llnl.gov

[4] F. J. DiSalvo, Science 285, 703-706 (1999).

[5] Spin Seebeck effect is also included here, which first converts the thermal difference into spin current and then into electric current, through the inverse spin Hall effect.

[6] L. E. Bell, Science 321, 1457-1461(2008).

[7] T. M. Tritt, Annu. Rev. Mater. Res. 41, 433-448 (2011).

[8] A. Shakouri, Annu. Rev. Mater. Res. 41, 399-431 (2011).

[9] S. B. Lang, Sourcebook of Pyroelectricity, Gordon \& Breach Science, London (1974).

[10] R W Whatmore, Rep. Prog. Phys. 49, 1335-1386 (1986).

[11] S. B. Lang, Phys. Today, 58, 31-36 (2005).

[12] Utilizing the ferro-paraelectric phase transition to generate electricity from temperature fluctuations also belongs to the pyroelectric effect.

[13] G. Sebald, D. Guyomar, and A. Agbossou, Smart Mater. Struct. 18, 125006 (2009).

[14] A. N. Morozovska E. A. Eliseev, G. S. Svechnikov, and S. V. Kalinin, J. Appl. Phys. 108, 042009 (2010).

[15] Y. Yang, W. Guo, K. C. Pradel, G. Zhu, Y. Zhou, Y. Zhang, Y. Hu, L. Lin, and Z. L. Wang, Nano Lett. 12, 2833-2838 (2012).

[16] Y. Yang, S. Wang, Y. Zhang, and Z. L. Wang, Nano Lett. 12, 6408-6413 (2012).

[17] Y. Yang, J. H. Jung, B. K. Yun, F. Zhang, K. C. Pradel, W. Guo, and Z. L. Wang, Adv. Mater. 24, 5357-5362 (2012).

[18] J. A. Rogers and U. Paik, Nature Nanotech. 5, 429435 (2010).

[19] T. J. Seebeck, Abh. Preuss. Akad. Wiss. p. 265, (1822-1823).

[20] D. Brewster, Edinburgh. J. Sci., 1, 208-215 (1824).

[21] A. Majumdar, MRS Bulletin 38, 947-954 (2013).

[22] T. C. Harman, P. J. Taylor, M. P. Walsh, B. E. LaForge, Science 297, 2229 (2002).

[23] M. S. Dresselhaus et al., Adv. Mater. 19, 1043 (2007).

[24] P. Reddy, S.-Y. Jang, R. A. Segalman, and A. Majumdar, Science 315, 1568 (2007).

[25] W. Lee et al., Nature 498, 209-212 (2013).

[26] H.-P. Breuer and F. Petruccione, The Theory of Open Quantum Systems (New York, 2002).

[27] H. Haug and A. P. Jauho, Quantum Kinetics in Transport and Optics of Semiconductors (Springer-Verlag, Berlin, 2008).

[28] S. M. Sze and K. K. Ng, Physics of Semiconductor Devices (Wiley, 2006).

[29] J. Singh, Smart Electronic Materials: Fundamentals and Applications (Cambridge University Press, 2005).

[30] The square-wave approximation is justified since the temperature relaxation timescale in metallic leads can be very fast, even up to picosecond. Thus, once the fluctuation period is much larger than the relaxation time in metallic leads, the fluctua- 
tion can be safely approximated as an abrupt change. Moreover, we checked the fluctuation with smooth change, e.g., a singlefrequent sine or cosine function. The results do not change qualitatively.

[31] S. Pancharatnam, Proc. Ind. Acad. Sci. A 44, 247 (1956).

[32] Y. Ben-Aryeh, J. Opt. B: Qauntum Semiclass. Opt. 6, R1
(2004).

[33] In Hermitian quantum mechanics, to avoid the cancellation of phases, at least three state change is necessary to form a closed loop with nonzero area in order to get the nonzero contribution.

[34] N. A. Sinitsyn, J. Phys. A: Math. Theor. 42, 193001 (2009).

[35] J. Ren, et al., Phys. Rev. Lett. 104, 170601 (2010); ibid., 108, 210603 (2012). 